\title{
A GENERALIZATION OF BRAUER CHARACTERS $\left({ }^{(}\right)$
}

\author{
BY \\ W. F. REYNOLDS \\ I. INTRODUCTION
}

1. Statement of results. The Brauer characters, or modular characters, of a finite group $G$ with respect to a prime $p$ are defined only on the $p$-regular elements of $G$. Brauer [1], [3] has overcome this limitation to some extent by using the Brauer characters of certain subgroups of $G$, in terms of which he has defined the generalized decomposition matrix $D$ of $G$. In this paper we study some complex-valued class-functions on $G$ which are closely related to these Brauer characters of subgroups. These functions, which we call $\phi$-functions, were defined by Brauer in [3, (7D)]. They behave in many ways like Brauer characters; for example, they have orthogonality relations ( $\$ 4)$ and they can be distributed among the $p$-blocks of $G(\S 9)$. In fact, our central motivating idea has been that the $\phi$-functions may be regarded as group-characters of a sort.

Explicitly, if $x$ is any $p$-element of $G$ and $\psi$ any irreducible Brauer character of the centralizer $C(x)$ of $x$ in $G$, there is a unique function $\phi$ defined on $G$ such that:

(a) $\phi$ is a class-function on $G$;

(b) $\phi(x y)=\psi(y)$ if $y$ is $p$-regular in $C(x)$;

(c) $\phi\left(x_{1} y\right)=0$ if $x_{1}$ is a $p$-element of $G$ which is not conjugate to $x$ and if $y$ is $p$-regular in $C\left(x_{1}\right)$.

We call these functions $\phi$ the $\phi$-functions of $G$ for the prime $p$. (Actually the definition of Brauer characters depends on the choice of a prime ideal divisor $\mathfrak{p}$ of $p$ in a suitable algebraic number field [6, p. 589]; accordingly, the $\phi$-functions also depend on $\mathfrak{p}$.) For $x=1, \phi$ is simply the Brauer character $\psi$ of $G$, if we extend the latter by giving it the value 0 on all $p$-singular elements (see the redefinition of Brauer characters in $\S 2$ ). Dually we define the $\Phi$-functions of $G$, starting from principal indecomposable characters $\Psi$ of $C(x)$; for $x=1$, these are the principal indecomposable characters of $G$.

$G$ has exactly $k$ distinct $\phi$-functions, where $k$ is the number of conjugate classes of $G$. If $\chi_{1}, \cdots, \chi_{k}$ are the (ordinary) irreducible characters of $G$, there are unique complex numbers $d_{i j}$ such that

$$
\chi_{i}(g)=\sum_{j=1}^{k} d_{i j} \phi_{j}(g)
$$

Presented to the Society, June 26, 1963; received by the editors August 13, 1964.

(1) This work has been supported in part by the National Science Foundation, through Harvard University, under grant number NSF-G-23833. 
for all $g \in G$. The square matrix $\boldsymbol{D}=\left(d_{i j}\right)$ is the generalized decomposition matrix of $G$ for $p$, as in [1] and [3], and the main theorem of [3] can be expressed by saying that $d_{i j}=0$ unless $\chi_{i}$ and $\phi_{j}$ are in the same $p$-block (see Theorem 9A below). This means that $\boldsymbol{D}$ breaks up completely into the square generalized decomposition matrices of the $p$-blocks of $G$.

If $G^{*}$ is any subgroup of $G$, the restriction $\phi \mid G^{*}$ to $G^{*}$ of a $\phi$-function $\phi$ of $G$ is a linear combination of the $\phi$-functions $\phi^{*}$ of $H$ with non-negative integral coefficients, or multiplicities (Theorem 5B); we say that $\phi \mid H$ contains $\phi^{*}$ if the multiplicity of $\phi^{*}$ in $\phi \mid H$ is positive. We can also define the function $\left(\phi^{*}\right)^{G}$ on $G$ induced by $\phi^{*}$ (see (2.5)), and obtain reciprocity formulas for induction and restriction ( $\$ 5)$.

Our principal results, which appear in parts III and IV, deal with normal subgroups of G. Part III is devoted to reduction theorems such as the following, which is proved in $\$ 7$ :

THEOREM 1A. Let $\tilde{\phi}$ be a $\phi$-function of a normal subgroup $H$ of $G$, and $I_{G}(\tilde{\phi})$ its inertial group in $G$ (see $\left.\S 2\right)$. Then the induction mapping $\phi^{\#} \rightarrow\left(\phi^{\#}\right)^{G}=\phi$ gives a one-to-one correspondence of the set of all $\phi$-functions $\phi^{\#}$ of $I_{G}(\tilde{\phi})$ such that $\phi^{\#} \mid H$ contains $\tilde{\phi}$ with the set of all $\phi$-functions $\phi$ of $G$ such that $\phi \mid H$ contains $\tilde{\phi}$. Furthermore, there is a precisely similar result for $\Phi$-functions.

In the case where $\tilde{\phi}$ is an irreducible Brauer character of $H$, this reduces to a reduction theorem of Clifford [4, Theorems 2 and 5]. Theorem $1 \mathrm{~A}$ gives no information on $\phi$-functions $\phi$ of $G$ such that $\phi \mid H=0$; such information is provided in Theorem $6 \mathrm{~A}$, which is essentially a generalization of Theorem $1 \mathrm{~A}$. These results for $\Phi$-functions appear to be new even in the case where $\tilde{\Phi}$ is a principal indecomposable character of $H$ (see [14]); there is probably a connection with part of the theorem of $[5, \S 2]$.

In part IV we apply Theorem $6 \mathrm{~A}$ to obtain the following reduction theorem for $p$-blocks:

THEOREM 1B. Let $B$ be a p-block of $G$, and let $H$ be any normal subgroup of $G$. Then there exists a group $G^{\prime}, H \subseteq G^{\prime} \subseteq G$, and a p-block $B^{\prime}$ of $G^{\prime}$, such that:

(a) the irreducible characters, the $\phi$-functions, and the $\Phi$-functions of $B^{\prime}$ are in one-to-one correspondence with their counterparts in $B$, all three of these correspondences being obtained by induction from $G^{\prime}$ to $G$;

(b) $B^{\prime}$ and $B$ have the same generalized decomposition matrix;

(c) the restrictions to $H$ of all irreducible characters, $\phi$-functions, and $\Phi$-functions of $B^{\prime}$ are linear combinations of the irreducible characters, $\phi$-functions, and $\Phi$-functions, respectively, of a single p-block $\widetilde{B}$ of $H$.

This theorem (see the end of \$11) serves to reduce questions involving blocks and normal subgroups to the situation where condition (c) holds. The special 
case of Theorem 1B for the ordinary decomposition matrix appeared as [13, Theorem 1], which was a generalization of a result due independently to Osima $[11$, Lemma 5] and Fong [8, (2B)]. Unfortunately I was unaware of [11], and therefore unable to give credit to Osima, while writing [13].

In view of Theorem $1 \mathrm{~B}$, it is natural to ask whether the block reductions in $[8,(2 D)]$ and $[13$, Theorem 6$]$, which preserve the ordinary decomposition matrix, also preserve the generalized decomposition matrix. This is not true for [8, (2D)]. In fact, let $H$ be the quaternion group, and $G$ its holomorph of order 24; then the three irreducible characters of degree 2 of $G$ make up a 3-block which provides a counterexample. On the other hand, the generalized decomposition matrix is preserved in a special case of [13, Theorem 6], namely when $G=D C_{G}(D)$ where $D$ is the defect group of $B$; this can be shown from the equations $[13,(18)]$. I hope to return to such questions in a subsequent paper.

2. Terminology. All groups referred to are finite. We work with respect to a fixed prime number $p$, writing block for $p$-block, etc. A group element is called $p$-regular, p-singular, or a p-element if its order is relatively prime to $p$, divisible by $p$, or a power of $p$, respectively. If $g=x y=y x$ with $x$ a $p$-element and $y$ $p$-regular, we call $x$ and $y$ the $p$-part and p-regular part, respectively, of $g$.

All Brauer characters, $\phi$-functions, and $\Phi$-functions are with respect to a fixed prime ideal divisor $\mathfrak{p}$ of $p$ (see $\S 1$ ), the same for all groups which appear. We depart from the usual definition of Brauer characters, according to which they are defined only on $p$-regular elements; instead, we say that the Brauer characters of a group $G$ are defined everywhere on $G$, having their usual values on the $p$-regular elements and the value zero on the $p$-singular elements.

If $\alpha$ is an element of the group-algebra $\Gamma(G)$ over a field $\Omega$, and if $g \in G$, we define

$$
\alpha^{g}=g^{-1} \alpha g \text {. }
$$

For fixed $\alpha$, the elements $\alpha^{g}, g \in G$, are said to form a family of $G$-conjugate (or $G$-associate) elements, and the group

$$
I_{G}(\alpha)=\left\{g \in G: \alpha^{g}=\alpha\right\}
$$

is called the inertial group (or stability group) of $\alpha$ with respect to $G$. If in particular $\alpha \in G$, we call $I_{G}(\alpha)$ the centralizer $C_{G}(\alpha)=C(\alpha)$ of $\alpha$ in $G$.

If $U$ is any subset of $G$, and $g \in G$, we set $U^{g}=\left\{u^{g}: u \in U\right\}$, and $N_{G}(U)=\left\{g \in G: U^{g}=U\right\}$. For any function $\sigma$ defined on $U$, let $\sigma^{g}$ be the function defined on $U^{g}$ such that

$$
\sigma^{g}\left(u^{g}\right)=\sigma(u), \quad u \in U .
$$

This definition is chosen so that $\left(\sigma^{g}\right)^{h}=\sigma^{g h}$ for $g, h \in G$. If $\sigma$ is a function defined on a normal subgroup of $G$, we call the functions $\sigma^{g}$ its $G$-conjugate functions, and define its inertial group $I_{G}(\sigma)$ by analogy with (2.2). 
If $\sigma$ is defined on $G$ and if $g \in G$, we also define a function $\sigma_{g}$ on $G$ by

$$
\sigma_{g}(u)=\sigma(g u), \quad u \in G .
$$

If $\tau_{1}$ and $\tau_{2}$ are complex-valued class-functions on $G$, their inner product is

$$
\left(\tau_{1}, \tau_{2}\right)_{G}=\left(\tau_{1}, \tau_{2}\right)=|G|^{-1} \sum_{g \in G} \tau_{1}(g) \overline{\tau_{2}(g)},
$$

where the bar denotes the complex-conjugate. The restriction of a class-function $\tau$ on $G$ to a subgroup $G^{*}$ of $G$ is denoted by $\tau \mid G^{*}$. Each class-function $\sigma$ on $G^{*}$ gives rise to an induced class-function $\sigma^{G}$ on $G$ defined $[6$, p. 266] by

$$
\sigma^{G}(u)=\left|G^{*}\right|^{-1} \sum_{g} \sigma\left(u^{g}\right), \quad u \in G,
$$

summed over all $g \in G$ such that $u^{g} \in G^{*}$.

\section{BASIC PROPERTIES}

3. The correspondence $i_{x, G}$. For each $p$-element $x$ of a group $G$, the p-section, or section, of $x$ in $G$ is the set $S_{G}(x)$ of all elements of $G$ whose $p$-part is $G$-con jugate to $x, G$ is the disjoint union of the sets $S_{G}(x)$ if $x$ ranges over a set of representatives of the conjugate classes of $p$-elements of $G$. In particular, $S_{G}(1)$ consists of all the $p$-regular elements of $G$.

We begin by establishing a correspondence $i_{x, G}$ between certain class-functions on $C(x)$ and on $G$, which will give rise to the $\phi$-functions and $\Phi$-functions. All class-functions here are complex-valued.

Lemma 3A. Let $x$ be a p-element of $G$; let $\sigma$ be any class-function on $C(x)$ which vanishes outside $S_{C(x)}(1)$. Then there is a unique class-function $\tau=i_{x, G} \sigma$ on $G$ which vanishes outside $S_{G}(x)$ such that

$$
\tau(x y)=\sigma(y), \quad y \in S_{C(x)}(1) .
$$

Proof. To show that $\tau$ is well defined, we need only show that if $x y_{1}$ and $x y_{2}$ are $G$-conjugate, where $y_{1}, y_{2} \in S_{C(x)}(1)$, then $y_{1}$ and $y_{2}$ are $C(x)$-conjugate. But if $\left(x y_{1}\right)^{g}=x y_{2}, g \in G$, equating $p$-parts and $p$-regular parts on both sides gives $x^{g}=x, y_{1}^{g}=y_{2}$, as required. (Cf. $[1, \S 1],[3, \S 3]$.)

This mapping $i_{x, G}$ is a one-to-one correspondence of the set of all class-functions on $C(x)$ which vanish outside $S_{C(x)}(1)$ with the set of all class-functions on $G$ which vanish outside $S_{G}(x)$. Each of these sets of functions is an algebra over the complex field under the usual operations, and $i_{x, G}$ is an algebra-isomorphism.

We now show that $i_{x, G}$ is an isometry with respect to the inner product of (2.5), and that it can be expressed in terms of induction (see (2.6)). 
LEMMA 3B. Let $x$ be a p-element of $G$; let $\sigma_{1}$ and $\sigma_{2}$ be class-functions on $C(x)$ which vanish outside $S_{C(x)}(1)$. Then

$$
\left(i_{x, G} \sigma_{1}, i_{x, G} \sigma_{2}\right)_{G}=\left(\sigma_{1}, \sigma_{2}\right)_{C(x)} .
$$

Proof. Set $\tau_{j}=i_{x, G} \sigma \sigma_{j}, j=1,2$. Then

$$
\begin{aligned}
\left(\tau_{1}, \tau_{2}\right) & =|G|^{-1} \sum_{g \in S_{G}(x)} \tau_{1}(g) \overline{\tau_{2}(g)}=|G|^{-1} \sum_{u} \sum_{y \in S_{C(x)}(1)} \tau_{1}\left((x y)^{u}\right) \overline{\tau_{2}\left((x y)^{u}\right)} \\
& =|G|^{-1}|G: C(x)| \sum_{y} \tau_{1}(x y) \overline{\tau_{2}(x y)}=|C(x)|^{-1} \sum_{y} \sigma_{1}(y) \overline{\sigma_{2}(y)}=\left(\sigma_{1}, \sigma_{2}\right),
\end{aligned}
$$

where $u$ ranges over a set of right coset representative of $C(x)$ in $G$.

LEMMA 3C. Let $x$ be a p-element of $G$, and let $\sigma$ be any class-function on $C(x)$ which vanishes outside $S_{C(x)}(1)$. Then $i_{x, G} \sigma=\left(\sigma_{x^{-1}}\right)$.

Proof. Since $\tau=\left(\sigma_{x-1}\right)^{G}$ is a class-function on $G$ and vanishes outside $S_{G}(x)$, we need only verify that it satisfies (3.1). But for $y \in S_{C(x)}(1)$,

$$
\tau(x y)=|C(x)|^{-1} \sum_{g} \sigma_{x-1}\left((x y)^{g}\right)=|C(x)|^{-1} \sum_{g} \sigma\left(x^{-1} x^{g} y^{g}\right),
$$

summed over all $g \in G$ such that $(x y)^{g} \in C(x)$. For such $g$, the $p$-part $x^{g}$ of $(x y)^{g}$ also lies in $C(x)$; hence $x^{-1} x^{g}$ is a $p$-element and commutes with $y^{g}$, so that it is the $p$-part of $x^{-1} x^{g} y^{g}$. Then $\sigma\left(x^{-1} x^{g} y^{g}\right)=0$ unless $x^{-1} x^{g}=1$, that is, unless $g \in C(x)$. Therefore

as required.

$$
\tau(x y)=|C(x)|^{-1} \sum_{c \in C(x)} \sigma\left(y^{c}\right)=\sigma(y)
$$

COROllaRY 3D. Under the assumptions of Lemma 3C, $i_{x, \mathrm{C}(x)^{\sigma}}=\sigma_{x^{-1}}$.

Remark. $S_{C(x)}(x)$ is a T.I. set in $G$ in the sense of [7]; hence Lemmas 3B and $3 \mathrm{C}$ could be obtained as corollaries to the lemma of [7].

4. The $\phi$-functions and $\Phi$-functions. Let $x$ be a $p$-element of $G, \psi$ an irreducible Brauer character of $C(x)$, and $\Psi$ the corresponding principal indecomposable character of $C(x)$. Then we say that $\phi=i_{x, G} \psi$ is a $\phi$-function of $G$ and that $\Phi=i_{x, G} \Psi$ is the $\Phi$-function of $G$ which corresponds to $\phi$.

All the $i_{x, G} \psi$ for a fixed $x$ are class-functions vanishing outside $S_{G}(x)$; their number is equal to the number of $p$-regular conjugate classes of $C(x)$, which in turn is equal to the number of classes of $G$-conjugate elements contained in $S_{G}(x)$ by the argument of Lemma 3A. The same $\phi$-functions are obtained if we replace $x$ by another $p$-element $x_{1}$ such that $S_{G}(x)=S_{G}\left(x_{1}\right)$ : for $x_{1}=x^{8}$ for some $g \in G$, and for each irreducible Brauer character $\psi$ of $C(x), \psi^{g}$ (see (2.3)) is an irreducible Brauer character of $(C(x))^{g}=C\left(x^{g}\right)$, and easily 
$i_{x^{g}, G} \psi^{g}=i_{x, G} \psi$ (cf. (5.5)). Therefore we call these the $\phi$-functions on the section $S_{G}(x)$.

Thus we obtain all $\phi$-functions of $G$ in the form $i_{x, G} \psi$ if we let $x$ range over a set of representatives of the conjugate classes of $p$-elements of $G$, and $\psi$ over all irreducible Brauer characters of $C(x)$; and the total number of $\phi$-functions is equal to the number $k$ of conjugate classes of $G$. The counterparts of all these statements for the $\Phi$-functions also hold. The $\phi$-functions on $S_{G}(1)$ are precisely the irreducible Brauer characters of $G$ (redefined as in $\S 2$ ), and the $\Phi$-functions on $S_{G}(1)$ are the principal indecomposable characters of $G$.

Let us denote the $\phi$-functions of $G$ by $\phi_{1}, \cdots, \phi_{k}$, and the $\Phi$-function corresponding to $\phi_{j}$ by $\Phi_{j}$. Since each $i_{x, G}$ is an isometry, and since two $\phi$-functions or $\Phi$-functions on different sections clearly have inner product zero, the orthogonality relations $[2, \S 3],[6, \S 84]$ for the Brauer characters of $C(x)$ imply orthogonality relations for the $\phi$ - and $\Phi$-functions, of the following form:

$$
\left(\phi_{j}, \Phi_{l}\right)=\delta_{j l}, \quad\left(\phi_{j}, \phi_{l}\right)=\gamma_{j l}, \quad\left(\Phi_{j}, \Phi_{l}\right)=c_{j l},
$$

where $j, l=1, \cdots, k$. Here $C=\left(c_{j l}\right)$ is the generalized Cartan matrix of $G$, defined as follows (cf. $[12, \S 11]$ ): if $\phi_{j}$ and $\phi_{l}$ are on different sections, $c_{j l}=0$; if $\phi_{j}=i_{x, G} \psi_{j}$ and $\phi_{l}=i_{x, G} \psi_{l}$, then $c_{j l}$ is the Cartan invariant of $C(x)$ corresponding to $\psi_{j}$ and $\psi_{l}$. Thus if the $\phi$-functions are arranged by sections $S_{G}(x)$, $\boldsymbol{C}$ has the Cartan matrices of the groups $C(x)$ as blocks along its diagonal, with zeros elsewhere, $I=\left(\delta_{j l}\right)$ is the identity matrix, and $C^{-1}=\left(\gamma_{j l}\right)$.

We can express these relations in matrix form. Let $\left\{g_{r}\right\}$ be a set of representatives of the conjugate classes of $G$; set $\phi=\left(\phi_{j}\left(g_{r}\right)\right), \boldsymbol{\Phi}=\left(\Phi_{j}\left(g_{r}\right)\right)$, and let $N$ be the diagonal matrix with entries $\left|G: C\left(g_{r}\right)\right|$. Then

$$
\phi N^{-1} \bar{\Phi}^{t}=I, \quad \phi N^{-1} \bar{\phi}^{t}=C^{-1}, \quad \Phi N^{-1} \bar{\Phi}^{t}=C,
$$

where the superscript $t$ denotes the transpose.

The $\phi$-function (or $\Phi$-functions) form a basis for the complex vector space of class-functions on $G$. By (4.1), the expressions of any class-function $\tau$ on $G$ in terms of these bases have the forms

$$
\tau=\sum_{j=1}^{k}\left(\tau, \Phi_{j}\right) \phi_{j}=\sum_{j=1}^{k}\left(\tau, \phi_{j}\right) \Phi_{j} .
$$

In particular, $\Phi_{j}=\Sigma_{l}\left(\Phi_{j}, \Phi_{l}\right) \phi_{l}=\Sigma_{l} c_{j l} \phi_{l}$, or

$$
\boldsymbol{\Phi}=\boldsymbol{C} \boldsymbol{\phi} \text {. }
$$

The equations (4.2) can be rewritten in the forms

$$
\overline{\boldsymbol{\Phi}}^{t} \phi=N, \quad \overline{\boldsymbol{\phi}}^{t} \boldsymbol{C} \phi=N, \quad \overline{\boldsymbol{\Phi}}^{t} C^{-1} \Phi=N,
$$

which yield the second set of orthogonality relations; for example, 


$$
\sum_{j=1}^{k} \bar{\Phi}_{j}(g) \phi_{j}(g)=|C(g)|, \quad g \in G .
$$

Now consider the (ordinary) irreducible characters $\chi_{1}, \cdots, \chi_{k}$ of $G$. By (4.3),

$$
\chi_{i}=\sum_{j=1}^{k} d_{i j} \phi_{j}, \quad \text { where } d_{i j}=\left(\chi_{i}, \Phi_{j}\right) \text {. }
$$

For any $p$-element $x$ and for $y \in S_{C(x)}(1)$,

$$
\chi_{i}(x y)=\sum_{j} d_{i j} \psi_{j}(y),
$$

where we sum only over those $j$ such that $\phi_{j}$ is on $S_{G}(x)$, and where $\phi_{j}=i_{x, G} \psi_{j}$. Comparing (4.8) with $[3,(3.2)]$, we see that the matrix $\boldsymbol{D}=\left(d_{i j}\right)$ is the generalized decomposition matrix of Brauer $[3, \S 7]$. Letting $\chi=\left(\chi_{i}\left(g_{r}\right)\right)$, we have $\chi=D \phi$. By the ordinary orthogonality relations, $\bar{\phi}^{t} \bar{D}^{t} D \phi=\bar{\chi}^{t} \chi=N=\bar{\phi}^{t} C \phi$, so that $[3,(3 A)]:$

$$
\bar{D}^{t} D=C
$$

Then $\boldsymbol{\Phi}=\boldsymbol{C} \phi=\bar{D}^{t} \boldsymbol{D} \phi=\bar{D}^{t} \chi$; that is,

$$
\boldsymbol{\Phi}=\overline{\mathbf{D}}^{t} \chi, \quad \text { or } \Phi_{j}=\sum_{i=1}^{k} d_{i j} \chi_{i} .
$$

5. Subgroups. We now consider the relationships between the $\phi$ - and $\Phi$-functions of $G$ and those of an arbitrary subgroup $G^{*}$ of $G$.

LEMMA 5A. If $x$ is a p-element of a subgroup $G^{*}$ of $G$, if $\sigma$ is a class-function on $C(x)$ which vanishes outside $S_{C(x)}(1)$, and if $\sigma^{*}$ is a class-function on $C_{G^{*}}(x)=C^{*}(x)$ which vanishes outside $S_{C^{*}(x)}(1)$, then

$$
\left(\left(i_{x, G} \sigma\right) \mid G^{*}, i_{x, G} \sigma^{*}\right)_{G^{*}}=\left(\sigma \mid C^{*}(x), \sigma^{*}\right)_{C *(x)} \text {. }
$$

Proof. We first see that $\left(i_{x, G} \sigma\right) \mid G^{*}$ takes on the same values as $i_{x, G *}\left(\sigma \mid C^{*}(x)\right)$ on $S_{G^{*}}(x)$ : since both are class-functions on $G^{*}$, we need only compare their values at elements $x y, y \in S_{C^{*}(x)}(1)$; but their definitions show that they both take on the value $\sigma(y)$ there. On the other hand, $i_{x, G} \sigma^{*}$ vanishes outside $S_{G *}(x)$. Therefore the left side of $(5.1)$ is equal to $\left(i_{x, G *}\left(\sigma \mid C^{*}(x)\right), i_{x, G *} \sigma^{*}\right) G^{*}$. But this equals the right side by Lemma 3B.

Let the $\phi$-functions of $G^{*}$ be denoted by $\phi_{m}^{*}$, with corresponding $\Phi$-functions $\Phi_{m}^{*}$. For any $\phi$-function $\phi_{j}$ of $G, \phi_{j} \mid G^{*}$ is a class-function on $G^{*}$. By (4.3),

$$
\phi_{j} \mid G^{*}=\sum_{m} a_{j m} \phi_{m}^{*}, \text { where } a_{j m}=\left(\phi_{j} \mid G^{*}, \Phi_{m}^{*}\right)_{G^{*}}
$$

We call $a_{j m}$ the multiplicity of $\phi_{m}^{*}$ in $\phi_{j} \mid G^{*}$.

THEOREM 5B. If $\phi_{j}$ is $a \phi$-function of $G$ and $\phi_{m}^{*}$ is $a \phi$-function of a subgroup $G^{*}$ of $G$, then the multiplicity $a_{j m}$ of $\phi_{m}^{*}$ in $\phi_{j} \mid G^{*}$ is a non-negative integer. 
Proof. Observe that $\phi_{m}^{*}$ is on $S_{G^{*}}(x)$ for some $p$-element $x$ of $G^{*}$. If $\phi_{j}$ is not on $S_{G}(x)$, clearly $a_{j \prime \prime \prime}=0$. If $\phi_{j}$ is on $S_{G}(x)$, then $\phi_{j}=i_{x, G} \psi, \phi_{m}^{*}=i_{x, G^{*}} \psi^{*}$ for certain irreducible Brauer characters $\psi, \psi^{*}$ of $C(x), C^{*}(x)$ respectively. By Lemma $5 \mathrm{~A}, a_{j_{m}}=\left(\psi \mid C^{*}(x), \Psi^{*}\right)$ where $\Psi^{*}$ is the principal indecomposable character corresponding to $\psi^{*}$. By (5.2), $a_{j m}$ is the multiplicity of $\psi^{*}$ in $\psi \mid C^{*}(x)$, and is a non-negative integer since $\psi$ and $\psi^{*}$ are Brauer characters.

We say that $\phi_{j} \mid G^{*}$ contains $\phi_{m}^{*}$ provided that $a_{j m}>0$. By the Frobenius reciprocity theorem $[6$, p. 271$], a_{j m}=\bar{a}_{j m}=\left(\Phi_{m}^{*}, \phi_{j} \mid G^{*}\right)=\left(\left(\Phi_{m}^{*}\right)^{G}, \phi_{j}\right)$. Then by (4.3),

$$
\left(\Phi_{m}^{*}\right)^{G}=\sum_{j} a_{j m} \Phi_{j}
$$

Dually, a similar argument shows that

$$
\Phi_{j} \mid G^{*}=\sum_{m} b_{j m} \Phi_{m}^{*}, \quad\left(\phi_{m}^{*}\right)^{G}=\sum_{j} b_{j m} \phi_{j},
$$

where $b_{j m}=\left(\Phi_{j} \mid G^{*}, \phi_{m}^{*}\right)=\left(\left(\phi_{m}^{*}\right)^{G}, \Phi_{j}\right)$ is a non-negative integer. These formulas generalize the reciprocity formulas for Brauer characters [6, p. 603].

Finally we consider conjugation. Let $x$ be a $p$-element of a subgroup $G^{*}$ of $G$, and let $\sigma^{*}$ be a class-function on $C_{G *}(x)=C^{*}(x)$ which vanishes outside $S_{C^{*}(x)}(1)$. Then for any $g \in G,\left(\sigma^{*}\right)^{g}$ is a class-function on $C_{\left(G^{*}\right)} g\left(x^{g}\right)$. For any $y \in S_{C^{*}(x)}(1)$,

$$
\left(i_{x, G} \sigma^{*}\right)^{g}\left(x^{g} y^{g}\right)=\left(i_{x, G} \sigma^{*}\right)(x y)=\sigma^{*}(y)=\left(\sigma^{*}\right)^{g}\left(y^{g}\right)=\left(i_{x^{g},(G *)}\left(\sigma^{*}\right)^{g}\right)\left(x^{g} y^{g}\right) .
$$

Therefore

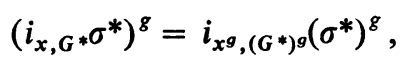

both sides being class-functions on $\left(G^{*}\right)^{g}$. In particular if $\sigma^{*}=\psi^{*}$ is an irreducible Brauer character of $C^{*}(x)$, then $\left(\psi^{*}\right)^{g}$ is an irreducible Brauer character of $C_{\left(G^{*}\right)} g\left(x^{g}\right)$, and (5.5) gives:

LEMMA 5C. If $G^{*}$ is a subgroup of $G$ and if $g \in G$, then the mapping $\phi^{*} \rightarrow\left(\phi^{*}\right)^{g}$ is a one-to-one correspondence of the set of all $\phi$-functions of $G^{*}$ with the set of all $\phi$-functions of $\left(G^{*}\right)^{g}$; and similarly for $\Phi$-functions.

COROLlaRY 5D. Any automorphism of a group induces a permutation of the set of all $\phi$-functions ( $\Phi$-functions) of the group.

\section{NORMAL SUBGROUPS}

6. Reduction theorem. Throughout part III, $H$ will be a fixed normal subgroup of $G$. For any $p$-element $x$ of $G$, set

$$
D(x)=C(x) \cap H .
$$


For any irreducible Brauer character $\theta$ of $D(x)$, let $E_{x}(\theta)$ be the set of all irreducible Brauer characters $\psi$ of $C(x)$ such that $\psi \mid D(x)$ contains $\theta$. Then for each $\psi \in E_{x}(\theta)$, a well-known theorem of Clifford [4, Theorem 1] says that

$$
\psi \mid D(x)=e_{\psi} \sum_{u} \theta^{u},
$$

where $e_{\psi}$ is a positive integer depending on $x$ and $\psi$, and $u$ ranges over a set of right coset representatives of $I_{C(x)}(\theta)$ in $C(x)$. Similarly let $E_{x}^{\#}(\theta)$ be the set of all irreducible Brauer characters $\psi^{\#}$ of $I_{C(x)}(\theta)$ such that $\psi^{\#} \mid D(x)$ contains $\theta$. By a reduction theorem of Clifford [4, Theorems 2 and 5], the induction mapping $\psi^{\#} \rightarrow\left(\psi^{\#}\right)^{C(x)}=\psi$ gives a one-to-one correspondence of $E_{x}^{\#}(\theta)$ and $E_{x}(\theta)$.

There is a corresponding result for principal indecomposable characters: if $\Theta, \Psi^{\#}$, and $\Psi$ are the principal indecomposable characters (of the appropriate groups) which correspond to $\theta, \psi^{\#}$, and $\psi$ in the preceding paragraph, then $\Psi \mid D(x)=f_{\psi} \Sigma_{u} \Theta^{u}$ for some positive integer $f_{\psi}$, and $\left(\Psi^{\#}\right)^{C(x)}=\Psi$. This reduction theorem has been stated in [14]; we shall use it only to show that the results for $\phi$-functions to which almost all of the present paper is devoted have analogues for $\Phi$-functions.

Let $F_{x}(\theta)=i_{x, G} E_{x}(\theta)$ be the set of all $\phi$-functions of $G$ of the form $i_{x, G} \psi$ with $\psi \in E_{x}(\theta)$. Similarly, with the group $I_{C(x)}(\theta) H$ in the role of $G$, let $F_{x}^{\#}(\theta)=i_{x, I_{C(r)}(\theta) H} E_{x}^{\#}(\theta)$. For this definition to make sense, we must show that

$$
I_{C(x)}(\theta)=C(x) \cap I_{C(x)}(\theta) H,
$$

the right side being the centralizer of $x$ in $I_{C(x)}(\theta) H$. But clearly $I_{C(x)}(\theta)$ is contained in this centralizer; and conversely if $c=u h, c \in C(x), u \in I_{C(x)}(\theta), h \in H$, then $u^{-1} c=h \in C(x) \cap H=D(x) \subseteq I_{C(x)}(\theta)$, and $c \in I_{C(x)}(\theta)$ as required.

We now prove our first main reduction theorem.

THEOREM 6A. Let $H$ be a normal subgroup of $G$, let $x$ be a p-element of $G$, and let $\theta$ be an irreducible Brauer character of $D(x)=C(x) \cap H$. Then the induction mapping $\phi^{\#} \rightarrow\left(\phi^{\#}\right)^{G}=\phi$ gives a one-to-one correspondence of $F_{x}^{\#}(\theta)$ with $F_{x}(\theta)$. Furthermore if $\phi^{\#} \in F_{x}^{\#}(\theta)$ and if $\Phi^{\#}$ and $\Phi$ are the $\Phi$-functions corresponding to $\phi^{\#}$ and $\phi$ respectively, then $\left(\Phi^{\#}\right)^{G}=\Phi$.

Proof. For any $\phi^{\#} \in F_{x}^{\#}(\theta), \phi^{\#}=i_{x, I c_{(x)}(\theta) H} \psi^{\#}$ for some $\psi^{\#} \in E_{x}^{\#}(\theta)$; and for any $\phi \in F_{x}(\theta), \phi=i_{x, G} \psi$ for some $\psi \in E_{x}(\theta)$. Then a one-to-one correspondence

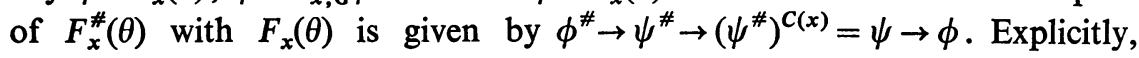

$$
\phi=\psi_{x^{-1}}^{G}=\left(\left(\left(\psi^{\#}\right)^{C(x)}\right)_{x-1}\right)^{G}=\left(\left(\psi_{x-1}^{\#}\right)^{C(x)}\right)^{G}=\left(\psi_{x-1}^{\#}\right)^{G}=\left(\left(\psi_{x-1}^{\#}\right)^{I_{C(. .)}(\theta) H}\right)^{G}=\left(\phi^{\#}\right)^{G} \text {, }
$$

where the third equality holds since $x^{-1}$ is in the center of $C(x)$. A similar calculation works for the $\Phi$-functions.

Observe that in the case $x=1$ this theorem specializes to the reduction theorems for irreducible Brauer characters and principal indecomposable characters which were used in its proof. 
The relationship between the different sets $F_{x}(\theta)$ is as follows:

LEMMA 6B. The set of all $\phi$-functions of $G$ is the disjoint union of the sets $F_{x}(\theta)$, where

(a) $x$ ranges over any set of representatives of the conjugate classes of p-elements of $G$, and

(b) for each $x, \theta$ ranges over any maximal set of non-C(x)-conjugate Brauer characters of $D(x)$.

Proof. By (6.2), the set of all irreducible Brauer characters of a fixed $C(x)$ is the disjoint union of the sets $E_{x}(\theta)$ for the $\theta$ in (b); hence the corresponding sets $F_{x}(\theta)$ give all the $\phi$-functions in $S_{G}(x)$.

The partitioning in Lemma $6 \mathrm{~B}$ is independent of the arbitrary choices of $x$ and $\theta$ made in fulfilling (a) and (b). In fact, by (5.5),

$$
F_{x^{g}}\left(\theta^{g}\right)=F_{x}(\theta), \quad g \in G .
$$

If $G^{*}$ is a subgroup of $G$ which contains $H$ and if $x$ is a $p$-element of $G^{*}$, we can define sets $E_{x}^{*}(\theta)$ and $F_{x}^{*}(\theta)$ analogous to $E_{x}(\theta)$ and $F_{x}(\theta)$, by taking $G^{*}$ in the role of $G$. The roles of $C(x)$ and $D(x)$ are played by $C^{*}(x)=C_{G} *(x)$ and $D(x)$ respectively. A connection between $F_{x}^{*}(\theta)$ and $F_{x}(\theta)$ is given by:

LEMMA 6C. If $\phi^{*} \in F_{x}^{*}(\theta)$, and if $\phi$ is a $\phi$-function of $G$ such that $\phi \mid G^{*}$ contains $\phi^{*}$, then $\phi \in F_{x}(\theta)$.

Proof. Clearly $\phi$ is on $S_{G}(x)$, so that $\phi=i_{x, G} \psi$ for some irreducible Brauer character $\psi$ of $C(x)$; and we know that $\phi^{*}=i_{x, G} \psi^{*}$ for some $\psi^{*} \in E_{x}^{*}(\theta)$. By Lemma $5 \mathrm{~A}$, the multiplicity of $\phi^{*}$ in $\phi \mid G^{*}$ is equal to that of $\psi^{*}$ in $\psi \mid C^{*}(x)$ (cf. the proof of Theorem 5B), so that $\psi \mid C^{*}(x)$ contains $\psi^{*}$. Since $\psi^{*} \mid H$ contains $\theta$ and since we are dealing with Brauer characters, $\psi \mid H$ contains $\theta$, so that $\psi \in E_{x}(\theta)$, which implies the result.

7. Some characterizations. Although $\S 6$ provides the background for almost all of part IV, Theorem $6 \mathrm{~A}$ is unsatisfactory in that the sets $F_{x}(\theta)$ and the groups $I_{C(x)}(\theta) H$ have been defined in terms of Brauer characters rather than $\phi$-functions. We now proceed to obtain characterizations of these objects in terms of $\phi$-functions, which will lead to Theorem 1A.

Again let $x$ be a $p$-element of $G$. If it happens that $x \in H$, then $\phi \mid H \neq 0$ for each $\phi$-function $\phi$ on $S_{G}(x)$, since $\phi(x) \neq 0$. On the other hand if $x \notin H$, then $\phi \mid H=0$ for each such $\phi$-function, since $S_{G}(x) \cap H$ is empty. In order to deal with the second possibility, we adjoin $x$ to $D(x)$ and to $H$ as follows: define the groups

$$
\hat{D}(x)=\langle x\rangle D(x), \quad \hat{H}(x)=\langle x\rangle H,
$$

where $\langle x\rangle$ is the cyclic group generated by $x$. If $x \in H$, then $\hat{D}(x)=D(x), \hat{H}(x)=H$; but if $x \notin H$, then $\hat{H}(x)$ need not even be normal in $G$. The motivation of this 
adjunction is that in the second case, the $\phi$-functions of $\hat{H}(x)$ take the place of some nonexistent $\phi$-functions of $H$. However, our results will be simpler and perhaps more natural in the first case.

Lemma 7A. There is a one-to-one correspondence $\theta \rightarrow \hat{\theta}$ of the set of all irreducible Brauer characters $\theta$ of $D(x)$ with the set of all irreducible Brauer characters $\hat{\theta}$ of $\hat{D}(x)$, such that $\hat{\theta} \mid D(x)=\theta$ and $\hat{\theta}$ vanishes outside $D(x)$.

Proof. Since $x$ centralizes $D(x), I_{\hat{D}(x)}(\theta)=\hat{D}(x)$. Since every factor set of the $p$-group $\hat{D}(x) \mid D(x)$ in an algebraically closed field of characteristic $p$ is equivalent to the trivial factor set (cf. [6, p. 359]), we obtain the desired one-to-one correspondence by one of Clifford's reduction theorems [4, Theorems 3 and 5]. Since all elements of $\hat{D}(x)$ outside $D(x)$ are $p$-singular, $\hat{\theta}$ vanishes on them.

By Lemma 7A, we see that if $\psi \in E_{x}(\theta)$ and $\hat{\theta} \mid D(x)=\theta$, then

$$
\psi \mid \hat{D}(x)=e_{\psi} \sum_{u} \hat{\theta}^{u}
$$

in the notation of (6.2).

As for $\hat{H}(x)$, we have $\hat{H}(x)=\langle x\rangle H=\langle x\rangle D(x) H=\hat{D}(x) H$, and

$$
\hat{D}(x)=C(x) \cap \hat{H}(x)=C_{\hat{H}(x)}(x)
$$

by an argument like the proof of (6.3). Hence to each irreducible character $\hat{\theta}$ of $\hat{D}(x)$ there corresponds a $\phi$-function $\hat{\phi}=i_{x, \hat{H}(x)} \hat{\theta}$ of $\hat{H}(x)$. Together with Lemma $7 \mathrm{~A}$, this yields a one-to-one correspondence $\hat{\imath}_{x}$ of the set of all irreducible Brauer characters $\theta$ of $D(x)$ with the set of all $\phi$-functions $\hat{\phi}$ of $\hat{H}(x)$ on $S_{\hat{H}(x)}(x)$, defined by setting $\hat{\phi}=\hat{\imath}_{x} \theta=i_{x, \hat{H}(x)} \hat{\theta}$ where $\hat{\theta} \mid D(x)=\theta$.

THEOREM 7B. Under the assumptions of Theorem 6A, $F_{x}(\theta)$ is the set of all $\phi$-functions $\phi$ of $G$ such that $\phi \mid \hat{H}(x)$ contains $\hat{\phi}$, where $\hat{\phi}=\hat{\imath}_{x} \theta$.

Proof. If a $\phi$-function $\phi$ of $G$ is not on $S_{G}(x)$, then $\phi$ is not in $F_{x}(\theta)$ and on the other hand $\phi \mid \hat{H}(x)$ vanishes on $S_{\hat{H}(x)}(x)$. Hence we need only consider $\phi$-functions $\phi$ on $S_{G}(x)$, say $\phi=i_{x, G} \psi$. As in the proof of Theorem 5B, the multiplicity of $\hat{\phi}$ in $\phi \mid \hat{H}(x)$ is equal to that of $\theta$ in $\psi \mid D(x)$; and by (7.2), the latter multiplicity is $e_{\psi}$ if $\psi \in E_{x}(\theta)$, that is if $\phi \in F_{x}(\theta)$, and 0 otherwise.

CoRollary 7C. If $\phi=i_{x, G} \psi \in F_{x}(\theta)$, the multiplicity of $\hat{i}_{x} \theta$ in $\phi \mid \hat{H}(x)$ is $e_{\psi}$.

CoRollary 7D. If $x \in H$ under the assumptions of Theorem $6 \mathrm{~A}, F_{x}(\theta)$ is the set of all $\phi$-functions $\phi$ of $G$ such that $\phi \mid H$ contains $\tilde{\phi}$, where $\tilde{\phi}=i_{x, H} \theta$.

THEOREM 7E. Under the assumptions of Theorem 6A, $\hat{H}(x)$ is a normal subgroup of $C(x) H$, and $I_{C(x)}(\theta) H=I_{C(x) H}(\hat{\phi})$ where $\hat{\phi}=\hat{\imath}_{x} \theta$.

Proof. The first statement is evident. Then $I_{C(x) H}(\hat{\phi})$ is defined, and any ele- 
ment of it has form $c h, c \in C(x), h \in H \subseteq I_{C(x) H}(\hat{\phi})$. Then $c \in C(x) \cap I_{C(x) H}(\hat{\phi})$, so that $I_{C(x) H}(\hat{\phi})=\left(C(x) \cap I_{C(x) H}(\hat{\phi})\right) H$. Thus it suffices to show that

$$
I_{C(x)}(\theta)=C(x) \cap I_{C(x) H}(\hat{\phi}) .
$$

But for $c \in C(x), \hat{\phi}^{c}=\left(i_{x, \hat{H}(x)} \theta\right)^{c}=i_{x, \hat{H}(x)} \hat{\theta}^{c}$ by $(5.5)$, so that $\hat{\phi}^{c}=\hat{i}_{x}\left(\theta^{c}\right)$. Then $\hat{\phi}^{c}=\hat{\phi}$ if and only if $\theta^{c}=\theta$, and this gives (7.5).

COROLLARY 7F. If $x \in H$ under the assumptions of Theorem $6 \mathrm{~A}, I_{C(x)}(\theta) H=I_{G}(\tilde{\phi})$ where $\tilde{\phi}=i_{x, H} \theta$.

By means of Corollaries 7D and 7F, we can rewrite the case $x \in H$ of Theorem $6 \mathrm{~A}$ to obtain Theorem $1 \mathrm{~A}$ of the introduction, which closely parallels the original Clifford reduction theorem. We may observe in this connection that if $\tilde{\Phi}$ is the $\Phi$-function of $H$ corresponding to $\tilde{\phi}$, then $\tilde{\Phi}^{g}$ corresponds to $\tilde{\phi}^{g}, g \in G$, whence $I_{G}(\tilde{\Phi})=I_{G}(\tilde{\phi})$. The following theorem, also for the case $x \in H$, generalizes the formula of Clifford which appears in (6.2).

THEOREM 7G. If $H$ is a normal subgroup of $G$ and if $\phi$ is a $\phi$-function of $G$ such that $\phi \mid H \neq 0$, then

$$
\phi \mid H=e_{\psi} \sum_{u} \tilde{\phi}^{u}
$$

where $\phi=i_{x, G} \psi \in F_{x}(\theta)$ for some p-element $x$ of $H, \tilde{\phi}=i_{x, H} \theta, e_{\psi}$ is as in (6.2), and $u$ ranges over a set of representatives of the right cosets of $I_{G}(\tilde{\phi})$ in $G$.

Proof. Since $\phi \mid H \neq 0, \phi \in S_{G}(x)$ for some $p$-element $x \in H$. Because $\phi$ is a class-function on $G$ which vanishes outside $S_{G}(x), \phi \mid H$ is a linear combination of functions of form $\Sigma_{u} \tilde{\phi}^{u}$, where $u$ ranges over a set of right coset representatives of $I_{G}(\tilde{\phi})$ in $G$, for a set of non-G-conjugate $\phi$-functions $\tilde{\phi}$ on $S_{H}(x)$. By Theorem 7B, we may suppose that $\phi \in F_{x}(\theta)$ where $\tilde{\phi}=i_{x, H} \theta$. This condition determines $\theta$ uniquely up to conjugation by elements of $C(x)$, by Lemma 6B; and by (5.5) it also determines $\tilde{\phi}$ up to conjugation, so that only one function $\Sigma_{u} \tilde{\phi}^{u}$ appears. The coefficient $e_{\psi}$ is given by Corollary 7C.

Parallel arguments lead to a corresponding formula $\Phi \mid H=f_{\psi} \Sigma_{u} \tilde{\Phi}^{u}$ for $\Phi$-functions.

8. Sections. In this section we characterize the group $C(x) H$ which appears in Theorem 7E, and then obtain a reduction theorem in terms of sections.

THEOREM 8A. If $H$ is a normal subgroup of $G$ and $x$ is a p-element of $G$, then $C(x) H=N_{G}\left(S_{\hat{H}(x)}(x)\right)$.

Proof. Under conjugation, each element of $C(x)$ fixes both $x$ and $\hat{H}(x)$ (by Theorem 7E), and hence fixes $S_{\widehat{H}(x)}(x)$; and clearly $H \subseteq \hat{H}(x) \subseteq N_{G}\left(S_{\hat{H}(x)}(x)\right)$. This gives one inclusion. Conversely, suppose that $g \in N_{G}\left(S_{\hat{H}(x)}(x)\right)$. Then 
$x^{g} \in S_{\hat{H}(x)}(x) ;$ that is, $x^{g}=x^{u}$ for some $u \in \hat{H}(x)$. Since $g u^{-1} \in C(x), g \in C(x) \hat{H}(x)$ $=C(x) H$.

THEOREM 8B. Let $H$ be a normal subgroup of $G$ and let $x$ be a p-element of $G$. Then the induction mapping $\phi^{0} \rightarrow\left(\phi^{0}\right)^{G}=\phi$ gives a one-to-one correspondence between the set of all $\phi$-functions $\phi^{0}$ of $C(x) H$ on $S_{C(x) H}(x)$ and the set of all $\phi$-functions $\phi$ of $G$ on $S_{G}(x)$. There is also a one-to-one correspondence $K^{0} \rightarrow K$ of the set of all $C(x) H$-conjugate classes $K^{0}$ in $S_{C(x) H}(x)$ and the set of all G-conjugate classes $K$ in $S_{G}(x)$, with $K^{0}=K \cap C(x) H$. Furthermore the value of $\phi^{0}$ on $K^{0}$ equals that of $\phi$ on $K$.

Proof. By their definitions, each irreducible Brauer character $\psi$ of $C(x)$ yields $\phi^{0}=\left(\psi_{x-1}\right)^{C(x) H}$ and $\phi=\left(\psi_{x-1}\right)^{G}$, giving the first correspondence. If $y$ ranges over a set of representatives of the $p$-regular conjugate classes of $C(x)$, the argument of Lemma $3 \mathrm{~A}$ shows that $x y$ ranges over a set of representatives for the classes $K$, and likewise for the classes $K^{0}$; this gives the second correspondence. Finally, $\phi(x y)=\psi(y)=\phi^{0}(x y)$.

Theorem $8 \mathrm{~B}$ is of interest principally as a counterpart to Theorem $11 \mathrm{~F}$ below, under the heuristic duality of sections and blocks [9]. $S_{\hat{H}(x)}(x)$ and its normalizer correspond to $B[\tilde{\eta}]$ and its inertial group; $\phi$-functions correspond to $\phi$-functions, conjugate classes to ordinary characters, and the preservation of values of $\phi$-functions to that of the generalized decomposition matrix.

\section{BLOCKS}

9. Blocks and $\phi$-functions. Let $G$ be any group. Let $\Omega$ be a field of characteristic $p$, in which all absolutely irreducible modular representations of all subgroups $G^{*}$ of $G$ can be written. For each such $G^{*}$, let $\Gamma\left(G^{*}\right)$ be the group-algebra of $G^{*}$ over $\Omega$, and $\Lambda\left(G^{*}\right)$ the center of $\Gamma\left(G^{*}\right)$. We consider these algebras as subalgebras of $\Gamma(G)$. The identity element of $\Gamma(G)$ is the sum of all the primitive idempotents $\eta$ of $\Lambda(G)$, and these are in a natural one-to-one correspondence $\eta \rightarrow B[\eta]$ with the blocks $B[\eta]$ (or, more accurately, $B_{G}[\eta]$ ) of $G$ (see $[2, \S 4], 6$, p. 604]).

If $x$ is any $p$-element of $G$, the primitive idempotents $\delta$ of $\Lambda(C(x))$ correspond in the same way to the blocks $B[\delta]$ (or $\left.B_{C(x)}[\delta]\right)$ of $C(x)$. Let $s_{x}$ be the mapping of $\Gamma(G)$ into $\Gamma(C(x))$ defined by

$$
s_{x}\left(\sum_{g \in G} \rho(g) g\right)=\sum_{c \in C(x)} \rho(c) c
$$

for all functions $\rho$ on $G$ with values in $\Omega$. Then $s_{x} \mid \Lambda(G)$ is a homomorphism of $\Lambda(G)$ into $\Lambda(C(x))$, by a theorem of Brauer [2,(7B)] applied to the $p$-subgroup $\langle x\rangle$. Then for each $\eta, s_{x}(\eta)$ is an idempotent or zero, and hence can be expressed uniquely as a sum of some of the idempotents $\delta$, which we call summands of $s_{x}(\eta)$; each $\delta$ is a summand of $s_{x}(\eta)$ for exactly one $\eta$. Observe that for any $\alpha \in \Gamma(G)$ and $g \in \mathbb{G}$, 


$$
\left(s_{x}(\alpha)\right)^{g}=s_{x^{g}}\left(\alpha^{g}\right) .
$$

We now distribute the $\phi$-functions of $G$ among the blocks of $G$ by defining that $\phi$ belongs to $B[\eta]$ if $\phi=i_{x, G} \psi$ where $\psi$ belongs to a block $B[\delta]$ of $C(x)$ such that $\delta$ is a summand of $s_{x}(\eta)$. This definition assigns just one block of $G$ to each $\phi$, and it is consistent with the usual definition for irreducible Brauer characters. It is independent of the choice of $x$ : for if we replace $x$ by $x^{g}, g \in G$, then $\phi=i_{x^{g}} \psi^{g}$ (cf. (5.5)), $\delta=g^{-1} \delta g$ (see (2.1)) is a primitive idempotent of $\Lambda\left(C\left(x^{g}\right)\right)$ which is a summand of $\left(s_{x}(\eta)\right)^{g}=s_{x^{g}}(\eta)$, and $\psi$ belongs to the block $B\left[\delta^{g}\right]$ of $C\left(x^{g}\right)$. We also say that each $\Phi$-function belongs to the same block as its corresponding $\phi$-function.

In this terminology, Brauer's theorem on generalized decomposition numbers (see [3] or [10] for proof), taken together with [3, (7E)], states:

THEOREM 9A. The generalized decomposition number $d_{i j}$ defined in (4.6) is zero unless $\chi_{i}$ and $\phi_{j}$ belong to the same block of $G$. Each block contains as many $\phi$-functions as irreducible characters.

Accordingly, we define the generalized decomposition matrix of $B[\eta]$ to be the square matrix $\left(d_{i j}\right)$ for $\chi_{i}$ and $\phi_{j}$ belonging to $B[\eta]$.

10. Normal subgroups. For the remainder of the paper, let $H$ be a fixed normal subgroup of $G$; we shall retain the notations of part III. The primitive idempotents $\tilde{\eta}$ of $\Lambda(H)$ correspond to the blocks $B[\tilde{\eta}]$ of $H$, and the primitive idempotents $\tilde{\delta}$ of $\Lambda(D(x))$ correspond to the blocks $B[\tilde{\delta}]$ of $D(x)$, where $x$ is any $p$-element of $G$ and $D(x)=C(x) \cap H$ as in (6.1).

Each primitive idempotent $\varepsilon$ of the commutative algebra $\Lambda(G) \cap \Lambda(H)$ is uniquely ex pressible as a sum of $\tilde{\eta}$ 's, and also as a sum of $\eta$ 's; each $\eta$ and each $\tilde{\eta}$ is a summand of just one $\varepsilon$. Similarly each primitive idempotent $\gamma$ of $\Lambda(C(x)) \cap \Lambda(D(x))$ is uniquely expressible as a sum of $\delta$ 's, and also as a sum of $\tilde{\delta}^{\prime}$ 's. These notations for dempotents will be used repeatedly in what follows.

$G$ has a permutation representation on the $\tilde{\eta}$ 's determined by the permutations $\tilde{\eta} \rightarrow \tilde{\eta}^{g}$ for $g \in G$, and correspondingly a representation $B[\tilde{\eta}] \rightarrow B\left[\tilde{\eta}^{8}\right]$ on the blocks of $H$. The ordinary characters of $B\left[\tilde{\eta}^{g}\right]$ are the $\chi^{g}$ where $\chi$ is an ordinary character of $B[\tilde{\eta}]$; and similarly for irreducible Brauer characters. For a given $\varepsilon$, the summands $\tilde{\eta}$ of $\varepsilon$ clearly make up an orbit of the first of these permutation representations, in other words a full set of $G$-conjugate $\tilde{\eta}$ 's. Therefore the idempotents $\varepsilon$ are the same as Fong's $\varepsilon_{i}[8, \mathrm{p}$. 266]. Analogously $C(x)$ is represented on the $\delta$ 's, and corresponding statements hold.

For each $\varepsilon$, let $\mathscr{T}[\varepsilon]$ (or $\mathscr{T}_{G, H}[\varepsilon]$ ) be the set of all blocks $B[\eta]$ of $G$ such that $\eta$ is a summand of $\varepsilon$, and let $\tilde{\mathscr{T}}[\varepsilon]$ be the set of all blocks $B[\tilde{\eta}]$ of $H$ such that $\tilde{\eta}$ is a summand of $\varepsilon$. These are the same as the sets $\mathscr{T}_{i}$ and $\mathscr{\mathscr { T }}_{i}$ of [8] and [12]. Let $T[\varepsilon]$ and $\tilde{T}[\varepsilon]$ be the sets of all $\phi$-functions in $\mathscr{T}[\varepsilon]$ and $\tilde{T}[\varepsilon]$ respectively; 
this defines partitionings of the sets of all $\phi$-functions of $G$ and of $H$. Correspondingly, for each $\gamma$ let $\mathscr{T}[\gamma]$ (or $\mathscr{T}_{C(x), D(x)}[\gamma]$ ) be the set of all blocks $B[\delta]$ of $C(x)$ such that $\delta$ is a summand of $\gamma$, and let $\tilde{T}[\gamma]$ be the set of all blocks $B[\tilde{\delta}]$ of $D(x)$ such that $\delta$ is a summand of $\gamma$; and define $T[\gamma]$ and $\tilde{T}[\gamma]$ accordingly.

LeMma 10A. Let $\theta$ be an irreducible Brauer character of $D(x)$, for the p-element $x$ of $G$. Then $\theta \in \tilde{T}[\gamma]$ if and only if $E_{x}(\theta) \subseteq T[\gamma]$.

Proof. By [8, (1B)], the restriction to $D(x)$ of any given irreducible character of $C(x)$ contains only irreducible characters of blocks in a single $\tilde{\mathscr{T}}[\gamma]$, and then the irreducible character is in a block of $\mathscr{T}[\gamma]$. Easily, this statement remains true if "irreducible character" is replaced by "irreducible Brauer character" (cf. $[13$, p. 18]); but this is the lemma.

Since $\varepsilon$ is a sum of $\eta$ 's, $s_{x}(\varepsilon)$ is an idempotent (or zero) in $\Lambda(C(x))$; and since $\varepsilon \in \Lambda(H)$, (9.1) shows that $s_{x}(\varepsilon)$ is in $\Lambda(C(x)) \cap \Lambda(D(x))$; therefore $s_{x}(\varepsilon)$ is uniquely expressible as a sum of $\gamma$ 's. We can obtain an expression for $s_{x}(\varepsilon)$ as a sum of $\delta$ 'sin two ways: firstly, $s_{x}(\varepsilon)$ is a sum of $\gamma$ 's, each of which is a sum of $\delta$ 's; secondly, $s_{x}(\varepsilon)$ is the sum of those $s_{x}(\eta)$ such that $\eta$ is a summand of $\varepsilon$, and each such $s_{x}(\eta)$ is a sum of $\delta$ 's. The fact that both these procedures must lead to the same summands $\delta$ of $s_{x}(\varepsilon)$ is used in proving the next lemma. Also $s_{x}(\varepsilon)$ can be expressed uniquely as a sum of $\tilde{\delta}$ 's, since it is a sum of $\gamma$ 's.

LeMma 10B. Let $\varepsilon$ be a primitive idempotent of $\Lambda(G) \cap \Lambda(H)$, and $\theta$ an irreducible Brauer character of $D(x)$. Then the following four conditions are equivalent:

(i) $\theta \in B[\tilde{\delta}]$ for some $\tilde{\delta}$ which is a summand of $s_{x}(\varepsilon)$;

(ii) $\theta \in \tilde{T}[\gamma]$ for some $\gamma$ which is a summand of $s_{x}(\varepsilon)$;

(iii) $F_{x}(\theta) \subseteq T[\varepsilon]$;

(iv) $F_{x}(\theta) \cap T[\varepsilon]$ is nonempty.

Proof. If (i) holds, then $\tilde{\delta}$ is a summand of some $\gamma$ which is a summand of $s_{x}(\varepsilon)$; this implies (ii).

If (ii) holds, suppose that $\phi \in F_{x}(\theta)$, that is $\phi=i_{x, G} \psi$ for some $\psi \in E_{x}(\theta)$. By Lemma $10 \mathrm{~A}, \psi \in T[\gamma]$; that is, $\psi$ is in a block $B[\delta]$ where $\delta$ is a summand of $\gamma$, and hence also of $s_{x}(\varepsilon)$. Then $\delta$ is a summand of some $s_{x}(\eta)$ such that $\eta$ is a summand of $\varepsilon$; but this means that $\phi \in B[\eta]$ and then $\phi \in T[\varepsilon]$, so that (ii) implies (iii).

Clearly (iii) implies (iv). Finally if (iv) holds, $\theta \in B[\tilde{\delta}]$ where $\tilde{\delta}$ is a summand of $s_{x}\left(\varepsilon_{1}\right)$ for some primitive idempotent $\varepsilon_{1}$ of $\Lambda(G) \cap \Lambda(H)$. Since (i) implies (iii), $F_{x}(\theta) \subseteq T\left[\varepsilon_{1}\right]$, whence $\varepsilon_{1}=\varepsilon$; thus (iii) implies (i).

Lemma 10B shows that the partitioning of the $\phi$-functions of $G$ into sets $F_{x}(\theta)$ (Lemma 6B) is a refinement of the partitioning into sets $T[\varepsilon]$.

Let $G^{*}$ be any subgroup of $G$ which contains $H$. For each primitive idempotent $\varepsilon^{*}$ of $\Lambda\left(G^{*}\right) \cap \Lambda(H)$, we can define sets $\mathscr{T}\left[\varepsilon^{*}\right]$ (that is, $\mathscr{T}_{G^{*}, H}\left[\varepsilon^{*}\right]$ ) and 
$\tilde{\mathscr{T}}\left[\varepsilon^{*}\right]$ of blocks, and sets $T\left[\varepsilon^{*}\right]$ and $\tilde{T}\left[\varepsilon^{*}\right]$ of $\phi$-functions, of $G^{*}$ and $H$ respectively, by applying the definitions of $\mathscr{T}[\varepsilon]$, etc., with $G^{*}$ in the role of $G$. Since $\Lambda\left(G^{*}\right) \cap \Lambda(H)=\left\{\alpha \in \Lambda(H): \alpha^{g}=\alpha\right.$ for all $\left.g \in G^{*}\right\} \subseteq \Lambda(G) \subseteq \Lambda(H)$, each of our idempotents $\varepsilon$ is in $\Lambda\left(G^{*}\right) \cap \Lambda(H)$, and hence is uniquely expressible as a sum of $\left(\varepsilon^{*}\right)$ 's.

Lemma 10C. Let $x$ be a p-element of $G^{*}$, and $\theta$ an irreducible Brauer character of $D(x)$. Then $F_{x}(\theta) \subseteq T[\varepsilon]$ if and only if $F_{x}^{*}(\theta) \subseteq T\left[\varepsilon^{*}\right]$ for some summand $\varepsilon^{*}$ of $\varepsilon$.

Proof. Recall that $F_{x}^{*}(\theta)$ is defined in the discussion preceding Lemma $6 \mathrm{C}$. By applying $[2,(7 \mathrm{~B})]$ to $G^{*}$, we see that $s_{x} \mid \Lambda\left(G^{*}\right)$ is a homomorphism of $\Lambda\left(G^{*}\right)$ into $\Lambda\left(C^{*}(x)\right)$, where $C^{*}(x)=C_{G^{*}}(x)$. Therefore for each $\varepsilon^{*}, s_{x}\left(\varepsilon^{*}\right)$ is an idempotent or zero in $\Lambda\left(C^{*}(x)\right) \cap \Lambda(D(x))$, which is contained in $\Lambda(C(x)) \cap \Lambda(D(x))$, and for each $\varepsilon, s_{x}(\varepsilon)$ is the sum of those $s_{x}\left(\varepsilon^{*}\right)$ such that $\varepsilon^{*}$ is a summand of $\varepsilon$. Now if $\theta \in B[\tilde{\delta}]$, Lemma $10 \mathrm{~B}$ shows that $F_{x}(\theta) \subseteq T[\varepsilon]$ if and only if $\tilde{\delta}$ is a summand of $s_{x}(\varepsilon)$, that is if and only if $\tilde{\delta}$ is a summand of $s_{x}\left(\varepsilon^{*}\right)$ for some summand $\varepsilon^{*}$ of $\varepsilon$; and then Lemma $10 \mathrm{~B}$, applied to $G^{*}$, gives the result.

11. Reduction theorem. We now center our attention on a fixed block $B[\tilde{\eta}]$ of $H$. The group $I_{G}(\tilde{\eta})$, which is also called the inertial group of $B[\tilde{\eta}]$ in $G$, will be denoted by $G^{\prime}$. (Of course this notation does not mean that $G^{\prime}$ is the commutator subgroup of $G$.) Let $\varepsilon$ be the unique primitive idempotent of $\Lambda(G) \cap \Lambda(H)$ such that $\tilde{\eta}$ is a summand of $\varepsilon$.

Since $H \subseteq G^{\prime} \subseteq G$, we can apply our results on groups $G^{*}$ to $G^{\prime}$, substituting primes for asterisks in the notations: for example, we set $C^{\prime}(x)=C_{G^{\prime}}(x)$. Since $\tilde{\eta}$ forms an orbit under the action of $G^{\prime}, \tilde{\eta}$ is itself a primitive idempotent $\varepsilon^{\prime}$ of $\Lambda\left(G^{\prime}\right) \cap \Lambda(H)$, and $\mathscr{T}[\tilde{\eta}]=\mathscr{T}_{G^{\prime}, H}[\tilde{\eta}]$ consists of $B[\eta]$ alone.

LEMMA 11A. If $x$ is a p-element of $G^{\prime}$ and if $\tilde{\delta}$ is a primitive idempotent of $\Lambda(D(x))$ which is a summand of $s_{x}(\tilde{\eta})$, then $I_{C(x)}(\tilde{\delta}) \subseteq C^{\prime}(x)$.

Proof. If $c \in I_{C(x)}(\tilde{\delta})$, then $c \in C(x)$ and $\tilde{\delta}^{c}=\tilde{\delta}$. Then $\tilde{\delta}$ is also a summand of $s_{x}\left(\tilde{\eta}^{c}\right)$ by $(9.2)$; hence $\tilde{\eta}^{c}=\tilde{\eta}$, so that $I_{C(x)}(\tilde{\delta}) \subseteq G^{\prime}$ as required.

If both $\theta$ and $\theta^{c}$ are irreducible Brauer characters of $B[\tilde{\delta}]$, then $\tilde{\delta}=\tilde{\delta}^{c}$. This yields the following corollaries.

COROLlaRY 11B. Under the assumptions of Lemma 11A, if $\theta$ is an irreducible Brauer character of $B[\tilde{\delta}]$, then $I_{C(x)}(\theta) \subseteq C^{\prime}(x)$.

COROLlary 11C. Under the assumptions of Lemma 11A, C(x)-conjugate Brauer characters of $B[\delta]$ are $C^{\prime}(x)$-conjugate.

THEOREM 11D. Let $H$ be a normal subgroup of $G$; let $\tilde{\eta}$ and $\varepsilon$ be primitive idempotents of $\Lambda(H)$ and $\Lambda(G) \cap \Lambda(H)$ respectively such that $\tilde{\eta}$ is a summand 
of $\varepsilon$. Then induction from $G^{\prime}=I_{G}[\tilde{\eta}]$ to $G$ gives a one-to-one correspondence of $T[\tilde{\eta}]$ with $T[\varepsilon]$.

Proof. Let $\phi^{\prime} \in T[\tilde{\eta}]$. By Lemmas 6B and 10B, applied to $G^{\prime}, \phi^{\prime} \in F_{x}^{\prime}(\theta) \subseteq T[\tilde{\eta}]$ for some $p$-element $x$ of $G^{\prime}$ and some irreducible Brauer character $\theta$ of $D(x)$. By Corollary $11 \mathrm{~B}$, the group $I_{C(x)}(\theta) H$ plays the same role in $G^{\prime}$ as in $G$; therefore Theorem $6 \mathrm{~A}$ for $G^{\prime}$ shows that $\phi^{\prime}=\left(\phi^{\#}\right)^{G^{\prime}}$ for some $\phi^{\#} \in F_{x}^{\#}(\theta)$. The same theorem for $G$ now shows that $\left(\phi^{\#}\right)^{G}$, which equals $\left(\phi^{\prime}\right)^{G}$, is a member $\phi$ of $F_{x}(\theta)$; while Lemma $10 \mathrm{C}$ shows that $F_{x}(\theta) \subseteq T[\varepsilon]$. Therefore induction gives a mapping $\phi^{\prime} \rightarrow \phi$ of $T[\tilde{\eta}]$ into $T[\varepsilon]$.

The part of the generalized decomposition matrix of $G^{\prime}$ belonging to the blocks in $\mathscr{T}[\tilde{\eta}]$ (cf. Theorem $9 \mathrm{~A}$ ) is the square matrix $\left(d_{i j}^{\prime}\right)$ given by the equations $\chi_{i}^{\prime}=\Sigma_{j} d_{i j}^{\prime} \phi_{j}^{\prime}$, where $\chi_{i}^{\prime}$ ranges over the irreducible characters in these blocks and $\phi_{j}^{\prime}$ ranges over the $\phi$-functions in these blocks, that is over $T[\tilde{\eta}]$. By $[13$, Theorem 1], the induction mapping $\chi_{i}^{\prime} \rightarrow\left(\chi_{i}^{\prime}\right)^{G}=\chi_{i}$ is a one-to-one correspondence of this set of irreducible characters and the set of all irreducible characters of the blocks of $\mathscr{T}[\varepsilon]$. Therefore inducing to $G$ yields $\chi_{i}=\Sigma_{j} d_{i j}^{\prime}\left(\phi_{j}^{\prime}\right)^{G}$. Since the $\chi_{i}$ are linearly independent and since the number of them is equal to the number of members of $T[\varepsilon]$ by Theorem $9 \mathrm{~A}$, we actually have a one-to-one mapping $\phi_{j}^{\prime} \rightarrow\left(\phi_{j}^{\prime}\right)^{G}=\phi_{j}$ of $T[\tilde{\eta}]$ onto $T[\varepsilon]$, as required. This also shows that $\left(d_{i j}^{\prime}\right)$ is the part of the generalized decomposition matrix $D$ of $G$ belonging to the blocks in $\mathscr{T}[\varepsilon]$.

COROLlaRY 11E. Under the assumptions of Theorem 11D, let $x$ be a p-element of $G$ which is not $G$-conjugate to any element of $G^{\prime}$. Then no $\phi$-function of $T[e]$ is on $S_{G}(x)$, and hence $s_{x}(\varepsilon)=0$.

THEOREM 11F. Let $H$ be a normal subgroup of $G$, and $B[\eta]$ any block of $G$. Let $\varepsilon$ be the primitive idempotent of $\Lambda(G) \cap \Lambda(H)$ of which $\eta$ is a summand; let $\tilde{\eta}$ be any primitive idempotent of $\Lambda(H)$ which is a summand of $\varepsilon$. Then there is a block $B\left[\eta^{\prime}\right]$ of $G^{\prime}=I_{G}(\tilde{\eta})$ such that induction from $G^{\prime}$ to $G$ gives one-to-one correspondences of the sets of (i) $\phi$-functions, (ii) irreducible characters, and (iii) $\Phi$-functions of $B\left[\eta^{\prime}\right]$ with the corresponding sets for $B[\eta]$. The blocks $B\left[\eta^{\prime}\right]$ and $B[\eta]$ have the same generalized decomposition matrix, and hence the same generalized Cartan matrix.

Proof. By Theorem 9A and, by [6, p. 609], the generalized decomposition matrix of a group determines the partitionings of the irreducible characters and the $\phi$-functions into blocks. Therefore the last sentence of the proof of Theorem 11D shows that in the induction mappings considered there, the irreducible characters and $\phi$-functions of $B[\eta]$ correspond to those of a certain block $B\left[\eta^{\prime}\right]$ in $\mathscr{T}[\eta]$. This yields everything except the statement about $\Phi$-functions; but by (4.9), 


$$
\left(\Phi_{j}^{\prime}\right)^{G}=\left(\sum_{i} \delta_{i j}^{\prime} \chi_{i}^{\prime}\right)^{G}=\sum_{i} \tilde{d}_{i j}^{\prime} \chi_{i}=\Phi_{j}
$$

Theorem 1B of the introduction is simply a restatement of Theorem 11F, except for statement (c) of Theorem 1B, which will be proved in $\$ 12$. Further information on the relationship between $B\left[\eta^{\prime}\right]$ and $B[\eta]$ appears in $[13$, Theorems 1 and 2]. It should be observed that the distributions of the $\phi$-functions of $B\left[\eta^{\prime}\right]$ and $B[\eta]$ among sections need not agree; an example of this phenomenon exists for $H$ of index 2 in the dihedral group $G$ of order 30 , with $p=5$.

12. Characterization of $T[\varepsilon]$. We conclude by giving a characterization of the sets $T[\varepsilon]$ in terms of $\phi$-functions, corresponding to the characterization of $F_{x}(\theta)$ in $\$ 7$.

LEMMA 12A. Let $\phi \in T[\varepsilon]$, and let $G^{*}$ be a subgroup of $G$ which contains $H$. Then $\phi \mid G^{*}$ is a linear combination (possibly zero) of members of the sets $T\left[\varepsilon^{*}\right]$ for the summands $\varepsilon^{*}$ of $\varepsilon$.

Proof. By Theorem 5B, we need only consider $\phi$-functions $\phi^{*}$ of $G^{*}$ such that $\phi \mid G^{*}$ contains $\phi^{*}$. For some $p$-element $x$ of $G^{*}$, irreducible Brauer character $\theta$ of $D(x)$, and primitive idempotent $\varepsilon^{*}$ of $\Lambda\left(G^{*}\right) \cap \Lambda(H), \phi^{*} \in F_{x}^{*}(\theta) \subseteq T\left[\varepsilon^{*}\right]$. By Lemma $6 \mathrm{C}, \phi \in F_{x}(\theta)$, so that $F_{x}(\theta) \subseteq T[\varepsilon]$ by Lemma $10 \mathrm{~B}$; and then Lemma 10C shows that $\varepsilon^{*}$ is a summand of $\varepsilon$.

COROLlARY 12B. If $\phi \in T[\varepsilon]$, then $\phi \mid H$ is a linear combination of members of the set $\tilde{T}[\varepsilon]$.

This corollary says that the restriction to $H$ of every $\phi$-function of the blocks in $\mathscr{T}[\varepsilon]$ contains only $\phi$-functions of blocks in $\tilde{\mathscr{T}}[\varepsilon]$ (cf. Theorem $7 \mathrm{G}$ ). The corresponding statements for ordinary functions and for $\Phi$-functions also hold, the first by $[8,(1 \mathrm{~B})]$, and the second by dualizing for $\Phi$-functions all the results on $\phi$-functions which lead up to Corollary 12B. When we apply these comments to $G^{\prime}$, we obtain statement (c) of Theorem $1 \mathrm{~B}$.

Because $\phi \mid H$ may vanish, Corollary 12B does not furnish a criterion for deciding whether $\phi \in T[\varepsilon]$. Instead we must use the groups $\hat{H}(x)$ of (7.1). If $\tilde{\eta}$ is a summand of $\varepsilon$, then since $\tilde{\eta}$ is a primitive idempotent of $\Lambda(H)$ and of $\Lambda\left(G^{\prime}\right) \cap \Lambda(H)$, it is also a primitive idempotent of $\Lambda(\hat{H})(x) \cap \Lambda(H)$, and therefore $\tilde{\eta}$ determines a set $\mathscr{T}_{\hat{\boldsymbol{H}}(x), H}[\tilde{\eta}]$ of blocks of $\hat{H}(x)$.

THEOREM 12C. Let $\phi$ be a $\phi$-function of $G$, and $\tilde{\eta}$ a fixed summand of $\varepsilon$. Then a necessary and sufficient condition that $\phi \in T[\varepsilon]$ is that there exists a p-element $x$ of $G^{\prime}$ such that $\phi \mid \hat{H}(x)$ contains some member of $T_{\hat{\boldsymbol{H}}(x), H}[\tilde{\eta}]$.

Proof. If $\phi \in T[\varepsilon]$, the proof of Theorem 11D shows that there exist a $p$-element $x \in G^{\prime}=I_{G}(\tilde{\eta})$ and an irreducible Brauer character $\theta$ of $D(x)$ such that 
$\phi \in F_{x}(\theta)$ while $F_{x}^{\prime}(\theta) \subseteq T[\tilde{\eta}]=T_{G^{\prime}, H}[\tilde{\eta}]$. By Theorem 7B, $\phi \mid \hat{H}(x)$ contains $\hat{\imath}_{x} \theta$. On the other hand if we apply Lemma $10 \mathrm{C}$ with $G^{\prime}$ in the role of $G$ and with $G^{*}=\hat{H}(x)$, we find that $F_{x}^{*}(\theta) \subseteq T_{\hat{H}(x), H}[\tilde{\eta}]$. Since $F_{x}^{*}(\theta)=\left\{\hat{\imath}_{x} \theta\right\}, \hat{\imath}_{x} \theta$ is the required member of $\mathscr{T}_{\hat{\boldsymbol{H}}(x), H}[\tilde{\eta}]$. The converse follows from Lemma 12A. Added in proof. In Theorem $12 \mathrm{C}, T_{\hat{\boldsymbol{H}}(x), H}[\tilde{\eta}]$ consists of a single block, by the following general lemma.

LEMMA 12D. If $H$ is a normal subgroup of $G$ and $G / H$ is a p-group, then each $T_{G, H}[\varepsilon]$ consists of a single block.

This is true since each summand $\eta$ of $\varepsilon$ in $\Lambda(G)$ is a linear combination of $p$-regular class sums $[6,(86.14)]$ and hence is in $\Lambda(G) \cap \Lambda(H)$, so that $\eta=\varepsilon$. An equivalent lemma (unpublished) has also been discovered by W. J. Wong.

\section{REFERENCES}

1. R. Brauer, On the connection between the ordinary and the modular characters of groups of finite order, Ann. of Math. (2) 42 (1941), 926-935.

2. —— Zur Darstellungstheorie der Gruppen endlicher Ordnung. I, Math. Z. 63 (1956), $406-444$.

3. —_ Zur Darstellungstheorie der Gruppen endlicher Ordnung. II, Math. Z. 72 (1959), $25-46$.

4. A. H. Clifford, Representations induced in an invariant subgroup, Ann. of Math. 38 (1937), 533-550.

5. S. B. Conlon, Twisted group algebras and their representations, J. Austral. Math. Soc. 4 (1964), 152-173.

6. C. W. Curtis and I. Reiner, Representation theory of finite groups and associative algebras, Interscience, New York, 1962.

7. W. Feit, Group characters. Exceptional characters, Proc. Sympos. Pure Math., pp. 67-70, Vol. 6, Amer. Math. Soc., Providence, R. I., 1962.

8. P. Fong, On the characters of p-solvable groups, Trans. Amer. Math. Soc. 98 (1961), 263-284.

9. K. Iizuka and T. Nakayama, A remark on orthogonality relations in finite groups, Nagoya Math. J. 20 (1962), 185-194.

10. H. Nagao, A proof of Brauer's theorem on generalized decomposition numbers, Nagoya Math. J. 22 (1963), 73-77.

11. M. Osima, On the representations of the generalized symmetric group. II, Math. J. Okayama Univ. 6 (1957), 81-97.

12. W. F. Reynolds, Modular representations of finite groups, Proc. Sympos. Pure Math., pp. 71-87, Vol. 6, Amer. Math. Soc., Providence, R. I., 1962. $15-32$.

13. - Blocks and normal subgroups of finite groups, Nagoya Math. J. 22 (1963),

14. - Principal indecomposable characters and normal subgroups, Abstract 63T-313, Notices Amer. Math. Soc. 10 (1963), 512.

TUFTS UNIVERSITY, MEDFoRd, MassachusetTS 